\title{
Coronary Stents in Patients with ST-Elevation Myocardial Infarction and Chronic Kidney Disease Undergoing Primary Percutaneous Coronary Intervention
}

\author{
Khurshid Ahmed, MD ${ }^{1,2}$, Myung Ho Jeong, MD1', Rabin Chakraborty, MD², Sumera Ahmed, MHM², \\ Young Joon Hong, MD1', Doo Sun Sim, MD¹, Keun Ho Park, MD¹, Ju Han Kim, MD¹, Youngkeun Ahn, MD1,

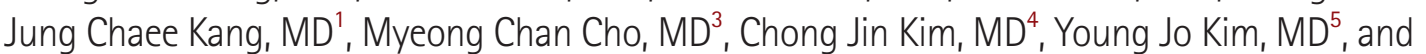 \\ Other Korea Acute Myocardial Infarction Registry Investigators \\ ${ }^{1}$ The Heart Center of Chonnam National University Hospital, Chonnam National University Research Institute of Medical Sciences, Gwangju, Korea
${ }^{2}$ Apollo Gleneagles Hospital, Kolkata, India, ${ }^{3}$ Department of Internal Medicine, Chungbuk National University Hospital, Chungju,
${ }^{4}$ Department of Internal Medicine, East West Neo Medical Center, Seoul, ${ }^{5}$ Department of Internal Medicine, Yeungnam University Hospital, Daegu, Korea
}

Background and Objectives: Chronic kidney disease (CKD) is associated with poor outcomes after percutaneous coronary intervention (PCI). We sought to compare different coronary stents used during primary PCI in patients with ST-elevation myocardial infarction (STEMI) and CKD. Subjects and Methods: We selected 2408 consecutive STEMI patients with CKD (estimated glomerular filtration rate $<60 \mathrm{~mL} / \mathrm{min} / 1.73 \mathrm{~m}$ ) undergoing primary $\mathrm{PCl}$ and divided them into 5 groups based on the type of stent implanted: 1) bare metal stent (BMS), 2) paclitaxeleluting stent (PES), 3) sirolimus-eluting stent (SES), 4) zotarolimus-eluting stent (ZES), or 5) everolimus-eluting stent (EES). The study endpoint was the number of major adverse cardiac events (MACE) at 12 months.

Results: There was no significant difference in the incidence of 12-month myocardial infarction, target lesion revascularization, or target vessel revascularization between stent groups; however, the overall rate of repeat revascularization differed significantly between groups. All-cause death differed significantly among the groups. The incidence of 12-month MACE in BMS, PES, SES, ZES, and EES was 8.3\%, 9.8\%, $8.6 \%, 5.5 \%$, and $2.6 \%$, respectively ( $p<0.001)$. Kaplan-Meier analysis did not show a significant differences in 12-month MACE-free survival among the groups (log-rank $p=0.076)$. This finding remained the same after adjusting for multiple confounders $(p=0.147)$.

Conclusion: Any of the 5 stents can be used to treat STEMI patients with CKD undergoing primary PCl; all have similar risk of 12-month MACE. This result is hypothesis-generating and warrants further evaluation with a long-term randomized study. (Korean Circ J 2012;42: 830-838)

KEY WORDS: Myocardial infarction; Stents; Angioplasty; Kidney failure, chronic.

\section{Introduction}

Patients with chronic kidney disease (CKD) present with accelerated atherosclerosis and higher rates of cardiovascular morbidity and mortality. ${ }^{1-3)}$ Furthermore, CKD is associated with adverse outcome following percutaneous coronary intervention $(\mathrm{PCl})^{455}$ which is probably related to endothelial dysfunction, inflammation, and platelet activation. After $\mathrm{PCl}$ using bare metal stent (BMS), the need for repeat revascularization remains high ${ }^{6)}$ even though the restenosis rate in mild to moderate CKD is not increased at 9 months. ${ }^{7)}$ Retrospective analyses have demonstrated that in the intermediate term, despite higher overall mortality and morbidity after $\mathrm{PCl}$, pa-

Received: June 20, 2012 / Revision Received: August 4, 2012 / Accepted: August 5, 2012

Correspondence: Myung Ho Jeong, MD, The Heart Center of Chonnam National University Hospital, Chonnam National University Research Institute of Medical Sciences, 42 Jebong-ro, Dong-gu, Gwangju 501-757, Korea

Tel: 82-62-220-6243, Fax: 82-62-228-7174, E-mail: myungho@chollian.net

- The authors have no financial conflicts of interest.

This is an Open Access article distributed under the terms of the Creative Commons Attribution Non-Commercial License (http://creativecommons.org/licenses/by-nc/3.0) which permits unrestricted non-commercial use, distribution, and reproduction in any medium, provided the original work is properly cited. 
tients with end stage renal disease ${ }^{8)}$ as well as those with milder degrees of $C{ }^{9}$ ) benefit from lower rates of target vessel revascularization (TVR) with drug-eluting stent (DES) versus BMS placement. Despite the high prevalence of coronary artery disease (CAD) among CKD patients, this patient population has been excluded consistently from the major clinical trials in cardiovascular medicine. ${ }^{10)}$

We sought to investigate which of the most commonly used stents best suit patients with moderate to severe CKD with ST-elevation myocardial infarction (STEMI) undergoing primary PCI. We compared 12-month clinical outcomes in 5 types of stents-BMS, paclitaxel-eluting stent (PES), sirolimus-eluting stent (SES), zotarolimuseluting stent (ZES) and everolimus-eluting stent (EES).

\section{Subjects and Methods}

This is a retrospective study that was carried out in the Heart Center of Chonnam National University Hospital, Gwangju, Korea. The permission to carry out the study was obtained from the hospital authorities (Institutional Review Board number 05-49, I-2008-01009) and written informed consent was obtained from all patients.

\section{Korea Acute Myocardial Infarction Registry}

The Korea Acute Myocardial Infarction Registry is a prospective, multicenter, observational registry designed to examine current epidemiology in hospital management and outcome of patients with acute myocardial infarction (AMI) in Korea, in commemoration of the 50th anniversary of the Korean Circulation Society. The registry included 52 community and university hospitals for primary PCI with 1-year clinical follow-up. Data was collected at each site by a welltrained study coordinator based procedures from a standardized protocol. ${ }^{11-13)}$

\section{Study population}

Patients with a suspected diagnosis of AMI were enrolled in the registry after admission to participating hospitals. Patients' medical documents were used to note the demographic data, clinical characteristics, and relevant laboratory results.

A total number of 2408 consecutive patients with ST-segment elevation MI and CKD undergoing primary PCl from Apr. 2006 through Sep. 2010 were selected for enrollment into this study. We studied patients' 12-month clinical outcomes (the follow-up rate was 70.5\%). CKD was defined as the estimated glomerular filtration rate (eGFR) $<60 \mathrm{~mL} / \mathrm{min} / 1.73 \mathrm{~m}^{2}$ calculated using the Modification of Diet in Renal Disease (MDRD) method. ${ }^{14)}$ Patients on hemodialysis or continuous ambulatory peritoneal dialysis, with cardiogenic shock and evidence of heart failure, were excluded from this study. Patients enrolled into the study were divided into 5 groups based on the type of stent implanted-BMS ( $n=292)$, PES $(n=381)$, SES $(n=420), Z E S(n=713)$, and EES $(n=602)$. A total of 2116 patients had undergone DES implantation.

\section{Definitions and clinical endpoints}

A final diagnosis of AMI was made according to the European Society of Cardiology/American College of Cardiology diagnostic criteria of AMI. ${ }^{15)}$ Patients with ST-segment elevation $\geq 1 \mathrm{~mm}$ in $\geq 2 \mathrm{ex}$ tremity electrocardiographic leads or $\geq 2 \mathrm{~mm}$ in $\geq 2$ contiguous precordial leads or new left bundle branch block on the admission electrocardiogram were defined as having STEMI. Hypertension was defined as systolic blood pressure $>140 \mathrm{~mm} \mathrm{Hg}$ and/or diastolic blood pressure $>90 \mathrm{~mm} \mathrm{Hg}$ at rest, at repeated measurements, or on treatment with anti-hypertensive medications. Diabetes was defined as having an established diagnosis of diabetes mellitus or the use of oral hypoglycemic agent or insulin to lower blood glucose levels. Hyperlipidemia was defined as total cholesterol level $>200 \mathrm{mg} / \mathrm{dL}$ or treatment with a lipid-lowering agent in this group of patients. CAD was defined as a history of $\mathrm{MI}$, of a revascularization procedure, or of obstructive CAD.

Peripheral blood samples were obtained using direct venipuncture. Blood samples were centrifuged, serum was removed, then serum samples were stored at a temperature of $-70^{\circ} \mathrm{C}$ until the assay could be performed to measure protein and sugar levels. Absolute creatine kinase-MB levels were determined by radioimmunoassay (Dade Behring, Inc., Miami, FL, USA). Cardiac-specific troponin I levels were measured by a paramagnetic particle, chemiluminescent immunoenzymatic assay (Beckham, Coulter, Inc., Fullerton, CA, USA). Twelvehour fasting serum levels of total cholesterol, triglyceride, and lowand high-density lipoprotein-cholesterol levels were measured by standard enzymatic methods. Blood samples for high-sensitivity Creactive protein (CRP) were obtained on admission, then analyzed turbidimetrically with sheep antibodies against human CRP; this has been validated against the Dade-Behring method.

Two-dimensional echocardiography was performed in all patients and left ventricular ejection fraction was assessed using a modification of Simpson's biplanar method. The morphology in coronary angiography was classified according to the criteria of the American College of Cardiology/American Heart Association (ACC/AHA). The degree of coronary flow was classified by Thrombolysis In Myocardial Infarction (TIMI) score. Renal function was assessed by eGFR, which was calculated using the MDRD method. ${ }^{14)}$ Presence of left main coronary artery stenosis was defined as luminal stenosis $\geq 50 \%$. Multivessel disease was defined as the presence of a lesion with $>50 \%$ diameter stenosis in a non-infarct related coronary artery. Successful PCl was defined as TIMI flow 3 with residual stenosis $\leq 50 \%$ in the infarct related artery. In-hospital complications included any of 
atrio-ventricular block, bradycardia, ventricular tachycardia/ventricular fibrillation, atrial fibrillation, cardiogenic shock, no re-flow, dissection, acute renal failure, metabolic acidosis/lactic acidosis, cerebrovascular event, or infection/sepsis. All patients were administered loading doses of aspirin $325 \mathrm{mg}$ and clopidogrel 300-600 mg before $\mathrm{PCl}$. Anticoagulation during $\mathrm{PCl}$ was performed according to the routine practices of each hospital. After the procedure, we prescribed 100-300 mg aspirin daily to patients on an indefinite basis.
For patients that underwent DES implantation, we prescribed clopidogrel for a full year after the procedure.

The clinical endpoint of this study was the occurrence of major adverse cardiac events (MACE), which included all-cause death, nonfatal myocardial infarction (MI), target lesion revascularization (TLR) and TVR over a 12-month follow-up period. Non-fatal MI was defined as the presence of clinical symptoms, electrocardiographic change, or abnormal imaging findings of Ml combined with an in-

Table 1. Baseline clinical and laboratory characteristics, and concomitant medications

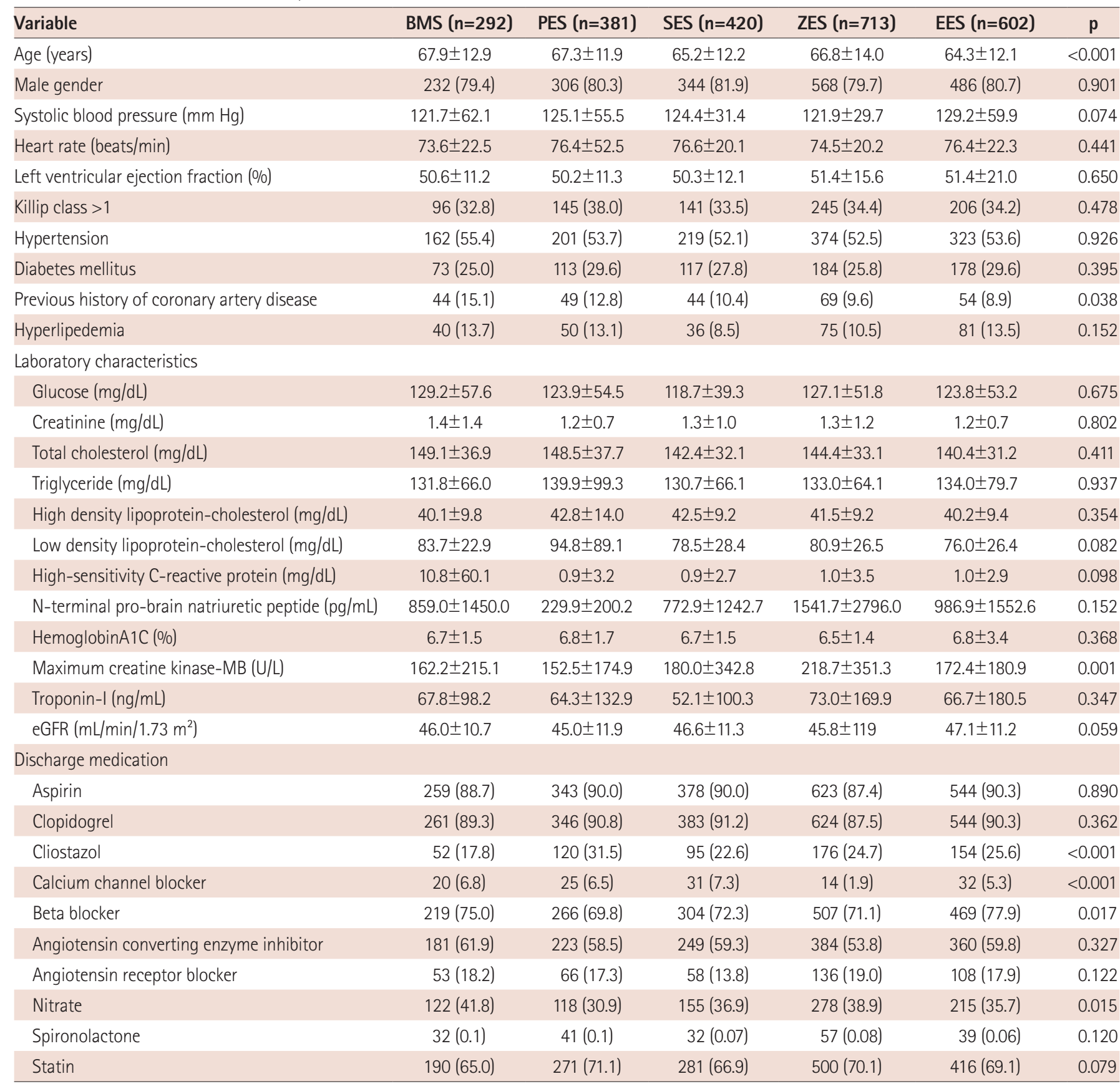

Data are expressed in mean \pm standard deviation, or number (percentage). BMS: bare metal stents, PES: paclitaxel-eluting stent, SES: sirolimus-eluting stent, ZES: zotarolimus-eluting stent, EES: everolimus-eluting stent, eGFR: estimated glomerular filtration rate 
crease in creatine kinase-MB fraction or troponin-I, at higher than the 99th percentile of the upper normal limit; this was not related to an interventional procedure. TVR defined as clinically-driven repeat revascularization of a lesion in the same epicardial vessel that had been treated during the index procedure at 12-month follow-up. TLR was defined as any revascularization of the target lesion due to restenosis or re-occlusion within $5 \mathrm{~mm}$ proximal/distal to the stent. All data were recorded on a standardized, electronic, web-based registry at http://www.kamir.or.kr.

\section{Statistical analysis}

Statistical Package for the Social Sciences (SPSS) 17.0 for windows (SPSS, Inc., Chicago, IL, USA) was used for all analyses. Continuous variables were presented as mean \pm standard deviation; comparisons were conducted by one-way analysis of variance test. Discrete variables were presented as percentages and frequencies; comparisons were conducted by chi-square statistics. A $p<0.05$ was considered statistically significant. Kaplan-Meier curves were constructed to examine the incidence of clinical endpoints. Cox proportional hazard analysis was performed in a step-wise manner to identify a model with independent predictive factors with determinat- ion of a hazard ratio and its 95\% confidence interval for each variable in the model. Covariates included in the model were age, low density lipoprotein-cholesterol (LDL-C), high-sensitivity CRP, N-terminal pro-brain natriuretic peptide, stent length, stent diameter, previous history of $C A D$, and use of medications, including beta-blocker, angiotensin receptor blocker, statin, pre-procedure TIMI flow grade $\mathrm{O}$, and use of glycoprotein Ill/Illa inhibitor. The number of 12-month MACE was relatively small in our study population; we limited the number of variables input into the model. The results are presented as adjusted hazard ratios with 95\% confidence intervals and $p$. We performed propensity score analysis (regression adjustment) to enable an even more rigorous adjustment for selection biases and confounding factors.

\section{Results}

A total number of 2408 consecutive patients with STEMI and CKD undergoing $\mathrm{PCl}$ were included in the present study. The mean age of the study population overall was $66.1 \pm 12.8$ years, the majority $(80.4 \%)$ were male. Mean stent length was $23.9 \pm 7.3 \mathrm{~mm}$ and mean stent diameter was $3.2 \pm 0.5 \mathrm{~mm}$. The mean number of stents used per

Table 2. Coronary angiographic characteristics and procedural results

\begin{tabular}{|c|c|c|c|c|c|c|}
\hline Variable & BMS $(n=292)$ & PES $(n=381)$ & SES $(n=420)$ & ZES $(n=713)$ & EES $(n=602)$ & $\mathrm{p}$ \\
\hline \multicolumn{7}{|l|}{ Intervened coronary artery } \\
\hline Left main & $2(0.7)$ & $7(1.8)$ & $9(2.1)$ & $13(1.8)$ & $8(1.3)$ & 0.570 \\
\hline Left circumflex & $26(8.9)$ & $32(8.3)$ & $47(11.2)$ & $65(9.1)$ & $62(10.3)$ & 0.653 \\
\hline Right coronary & $158(54.1)$ & $169(44.4)$ & $130(30.9)$ & $325(45.6)$ & $238(39.5)$ & $<0.001$ \\
\hline $\begin{array}{l}\text { Pre-Thrombolysis in Myocardial Infarction } \\
\text { grade } 0 \text { flow }\end{array}$ & $174(59.6)$ & $199(52.2)$ & $211(50.2)$ & $436(61.2)$ & $311(51.6)$ & 0.001 \\
\hline $\begin{array}{l}\text { Post-Thrombolysis in Myocardial Infarction } \\
\text { grade } 3 \text { flow }\end{array}$ & $292(100)$ & $381(100)$ & $420(100)$ & 712 (99.86) & 600 (99.67) & $<0.483$ \\
\hline Stent diameter (mm) & $3.3 \pm 0.6$ & $3.2 \pm 0.4$ & $3.0 \pm 0.4$ & $3.1 \pm 0.4$ & $3.1 \pm 0.5$ & $<0.001$ \\
\hline Pre-PCl diameter stenosis (\%) & $83.1 \pm 20.4$ & $84.3 \pm 16.9$ & $88.9 \pm 13.0$ & $90.9 \pm 13.7$ & $90.0 \pm 15.7$ & $<0.001$ \\
\hline Post-PCl diameter stenosis (\%) & $11.7 \pm 10.0$ & $12.6 \pm 7.8$ & $13.9 \pm 14.1$ & $11.2 \pm 8.1$ & $11.3 \pm 13.4$ & 0.051 \\
\hline Glycoprotein Ilb/IIla inhibitor & $66(22.6)$ & $65(17.0)$ & $58(13.8)$ & $97(13.6)$ & 77 (12.8) & 0.002 \\
\hline Thrombus aspiration & $73(25.0)$ & $138(36.2)$ & $138(32.8)$ & $251(35.2)$ & $180(29.9)$ & 0.003 \\
\hline $\begin{array}{l}\text { Percutaneous coronary intervention } \\
\text { success rate }\end{array}$ & $283(96.9)$ & $367(96.3)$ & $387(92.1)$ & $693(97.2)$ & $580(96.3)$ & 0.001 \\
\hline In-hospital complications & $53(18.1)$ & $40(10.5)$ & $40(9.5)$ & $66(9.2)$ & $53(8.8)$ & $<0.001$ \\
\hline
\end{tabular}

Data are expressed in mean \pm standard deviation, or number (percentage). BMS: bare metal stent, PES: paclitaxel-eluting stent, SES: sirolimus-eluting stent, ZES: zotarolimus-eluting stent, EES: everolimus-eluting stent, PCI: percutaneous coronary intervention 
vessel was 1.4 \pm 0.7 .

\section{Baseline clinical characteristics, coronary angiographic findings and procedural results}

Baseline clinical and laboratory characteristics, along with concomitant medications of the 5 groups, are presented in Table 1. Mean age was highest in the BMS group, followed by PES, ZES, SES, with the lowest mean age in the EES group $(p<0.001)$. The prevalence of previous history of CAD was highest in the BMS group, followed by the PES, SES, ZES and lowest in EES group ( $p=0.038)$. Creatine kinase-MB was highest in ZES group, followed by SES, EES, BMS and $P E S$ groups ( $p=0.001)$. The use of cilostazol was highest in the PES ( $p<$ $0.001)$, calcium channel blocker use was highest in the SES $(p<0.001)$, beta blocker use was highest in the EES $(p=0.017)$ and nitrate in the BMS group ( $p=0.015)$.

Coronary angiographic characteristics and procedural results of the 5 groups are presented in Table 2. Left anterior descending artery involvement was highest in SES group, followed by EES, PES, ZES, and lowest in BMS group $(p<0.001)$. Right coronary involvement was highest in BMS, then ZES, PES, EES and SES ( $p=0.001)$. Pre-PCI TIMI grade 0 flow was highest in the ZES group, followed by the BMS, PES, EES and SES ( $p=0.001)$. Mean stent length was highest in
PES, followed by SES, ZES, EES and BMS groups ( $p<0.001)$. Mean stent diameter was highest in the BMS and lowest in SES group ( $p<$ 0.001). Percent diameter stenosis before PCI was highest in the ZES, followed by EES, SES, PES, and BMS groups ( $p<0.001)$. Use of glycoprotein IIb/Illa inhibitor was highest in BMS and lowest in EES groups ( $p=0.002$ ), whereas use of thrombus aspiration device was highest in the PES and lowest in the BMS groups. PCl success rate was highest in the ZES group, followed by the BMS group and then almost similar in the PES and EES groups, and lowest in the SES group. In-hospital complications were highest in BMS group, followed by PES, SES, ZES, and lowest in EES group. Other coronary angiographic characteristics did not show statistically meaningful differences among the groups.

Twelve-month clinical outcomes are presented in Table 3. The incidence of 12-month MACE decreased from PES, SES, BMS, ZES to EES groups ( $9.8 \%$ vs. $8.6 \%$ vs. $8.3 \%$ vs. $5.5 \%$ vs. $2.6 \%, p<0.001$ ). Incidence of 12-month all-cause death, which was attributable to cardiac death, was highest in the BMS group and lowest in the EES group ( $\mathrm{p}=0.002)$. However, it is noteworthy that non-fatal MI, TVR, TLR, and stent thrombosis were not statistically significant among the groups. One acute event of stent thrombosis occurred in the BMS group, 7 sub-acute stent thromboses (2 in BMS, 2 in PES, 1 in SES,

Table 3. One-month and twelve-month clinical outcomes

\begin{tabular}{|c|c|c|c|c|c|c|}
\hline Outcome variable & BMS & PES & SES & ZES & EES & \\
\hline At 1-month & $\mathrm{N}=\mathbf{2 1 7}$ & $\mathrm{N}=\mathbf{2 8 2}$ & $\mathrm{N}=335$ & $\mathrm{~N}=432$ & $\mathrm{~N}=419$ & 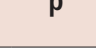 \\
\hline Major adverse cardiac event & $12(5.5)$ & $6(2.1)$ & $2(0.8)$ & $9(2.0)$ & $5(1.2)$ & 0.001 \\
\hline Cardiac death & $8(3.7)$ & $3(1.0)$ & $1(0.4)$ & $5(1.2)$ & $0(0)$ & $<0.001$ \\
\hline Non-cardiac death & $3(1.4)$ & $0(0)$ & $1(0.4)$ & $2(0.5)$ & $2(0.5)$ & 0.223 \\
\hline Target vessel & $0(0)$ & $0(0)$ & $0(0)$ & $0(0)$ & $0(0)$ & 0.999 \\
\hline Target lesion & $0(0)$ & $1(0.3)$ & $0(0)$ & $0(0)$ & $0(0)$ & 0.256 \\
\hline Non-target vessel & $1(0.5)$ & $2(0.7)$ & $0(0)$ & $1(0.2)$ & $2(0.5)$ & 0.670 \\
\hline At 12-month & $\mathrm{N}=217$ & $\mathrm{~N}=\mathbf{2 8 4}$ & $\mathrm{N}=342$ & $\mathrm{~N}=433$ & $\mathrm{~N}=421$ & \\
\hline Non-cardiac death & $3(1.4)$ & $2(0.7)$ & $2(0.8)$ & $6(1.4)$ & $3(0.7)$ & 0.066 \\
\hline Non-fatal myocardial infarction & $1(0.5)$ & $2(0.7)$ & $1(0.4)$ & $1(0.9)$ & $1(0.2)$ & 0.786 \\
\hline Repeat revascularization & $5(2.3)$ & $17(6.0)$ & $16(4.7)$ & $11(2.5)$ & $7(1.7)$ & 0.001 \\
\hline Target vessel & $0(0)$ & $3(1.0)$ & $4(1.2)$ & $4(0.9)$ & $1(0.2)$ & 0.314 \\
\hline Target lesion & $4(1.8)$ & $8(2.8)$ & $7(2.0)$ & $5(1.2)$ & $1(0.2)$ & 0.053 \\
\hline Non-target vessel & $1(0.5)$ & $6(2.1)$ & $5(1.5)$ & $2(0.5)$ & $5(1.2)$ & 0.184 \\
\hline Stent thrombosis & $5(2.3)$ & $2(0.7)$ & $3(0.8)$ & $3(0.7)$ & $2(0.4)$ & 0.279 \\
\hline
\end{tabular}

Data are expressed in number (percentage). BMS: bare metal stent, PES: paclitaxel-eluting stent, SES: sirolimus-eluting stent, ZES: zotarolimus-eluting stent, EES: everolimus-eluting stent 
1 in ZES, and 1 in EES groups), 4 late stent thromboses (1 in SES, 2 in ZES, and 1 in EES) and 3 very late stent thromboses (2 in BMS and 1 in SES groups) occurred. The average rate of $\mathrm{PCl}$ success was $95.9 \%$.

Kaplan-Meier survival analysis demonstrated insignificant differences in freedom from MACE among the 5 groups $(\log$-rank $p=$

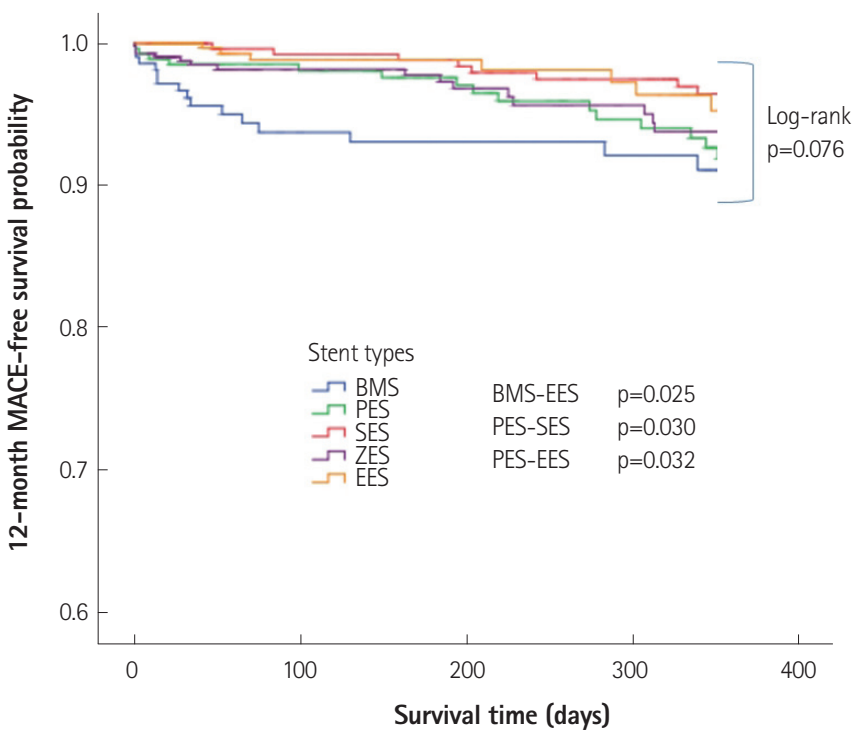

Fig. 1. Kaplan-Meier curves for 12-month MACE-free survival for patients with STEMI and CKD undergoing primary PCI treated with 5 commonly used stent types. MACE: major adverse cardiac events, STEMI: ST-elevation myocardial infarction, CKD: chronic kidney disease, $\mathrm{PCl}$ : percutaneous coronary intervention, BMS: bare metal stent, PES: paclitaxel-eluting stent, SES: sirolimus-eluting stent, ZES: zotarolimus-eluting stent, EES: everolimus-eluting stent.

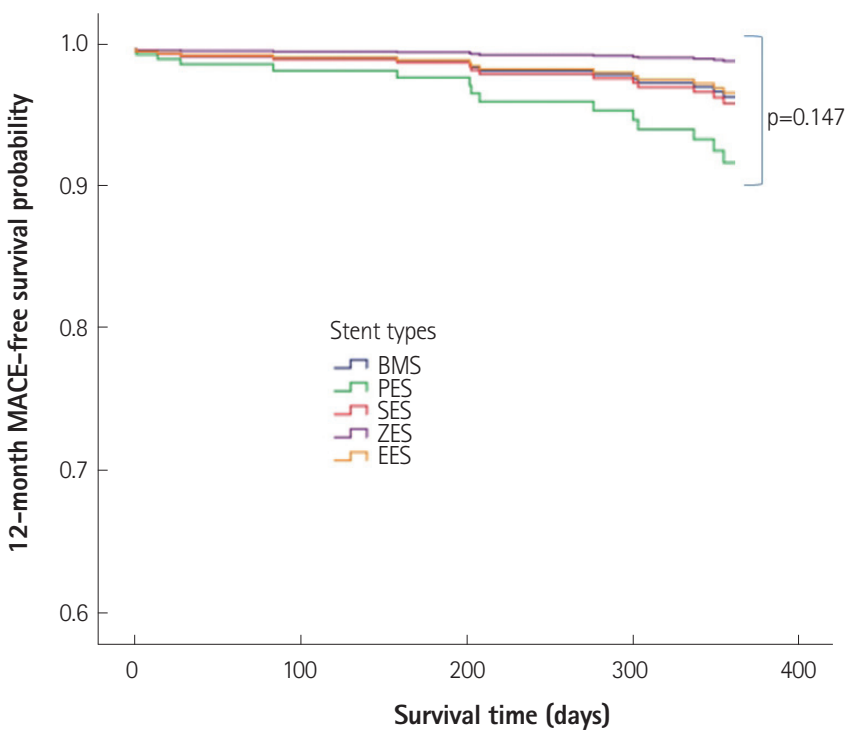

Fig. 2. Adjusted 12-month MACE-free survival in patients with STEMI and CKD undergoing primary $\mathrm{PCl}$ using 5 types of commonly used stents. MACE: major adverse cardiac events, STEMI: ST-elevation myocardial infarction, CKD: chronic kidney disease, PCl: percutaneous coronary intervention, BMS: bare metal stent, PES: paclitaxel-eluting stent, SES: sirolimus-eluting stent, ZES: zotarolimus-eluting stent, EES: everolimus-eluting stent.
0.076) (Fig. 1). A pair-wise comparison revealed significant differences between BMS and EES ( $p=0.025)$, PES and SES $(p=0.030)$, and between PES and EES ( $p=0.032$ ). Cox proportional hazard analysis revealed no statistical differences in the incidence of MACE when adjusted for age and gender ( $p=0.130)$, and also when adjusted for multiple confounders ( $p=0.147$ ) (Fig. 2). MACE at 12 months did not differ significantly among groups despite propensity score adjustment using multinomial logistic or Cox model $(p=0.145)$ (Fig. 3).

\section{Discussion}

The rate of cumulative MACE differed significantly among the groups, mainly driven by the risk of cardiac death. However, after adjustment for multiple confounders, the risk of MACE was similar among the groups. We found that any of the 5 commonly used stents can be used to treat STEMI patients with CKD undergoing primary $\mathrm{PCl}$, with a similar risk of MACE.

Most studies of cardiovascular outcomes have found that a breakpoint for the development of contrast-induced nephropathy, acute, or subacute stent thrombosis, later restenosis, recurrent myocardial infarction, diastolic/systolic congestive heart failure, and cardiovascular death occurs below an eGFR of $60 \mathrm{~mL} / \mathrm{min} / 1.73 \mathrm{~m}^{2}$, which roughly corresponds to a serum creatinine level higher than $1.5 \mathrm{mg} / \mathrm{dL}$ in the general population. ${ }^{16) 17)} \mathrm{A}$ study by Bonello et al. ${ }^{18)}$ showed a graded increase in serious in-hospital complications (death, vascular complications, need for transfusion, acute renal failure, MACE), 1-month events (death, repeat MI, TLR, MACE), and 1-year outcomes

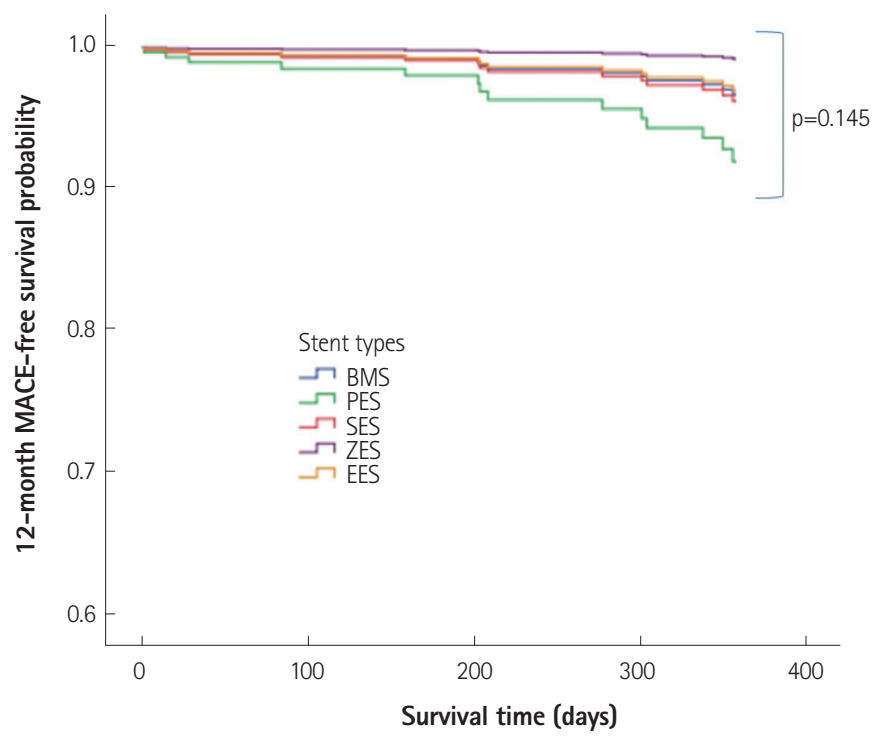

Fig. 3. Propensity adjusted 12-month MACE-free survival in patients with STEMI and CKD undergoing primary PCl using 5 types of commonly used stents. MACE: major adverse cardiac events, STEMI: ST-elevation myocardial infarction, CKD: chronic kidney disease, PCl: percutaneous coronary intervention, BMS: bare metal stent, PES: paclitaxel-eluting stent, SES: sirolimus-eluting stent, ZES: zotarolimus-eluting stent, EES: everolimus-eluting stent. 
(death, MI, TLR, MACE) as renal function declined. In spite of these poor outcomes, the use of $\mathrm{PCl}$ is associated with superior long-term survival when compared with medical therapy alone in the management of acute coronary syndrome in CKD patients. ${ }^{19)}$ Therefore, we thought to analyze the most commonly used stents in patients with moderate to severe CKD.

The frequency of clinical adverse events was also higher according to the stages of CKD. Hachinohe et al. ${ }^{20)}$ demonstrated when patients with non-ST-segment elevation MI have severe CKD, the conservative or deferred invasive strategy with prescription of cardio-protective medication and prevention of further deterioration in renal function should be considered. Although the use of new interventional devices, including stents, improved immediate procedural success rates, CKD patients had higher long-term mortality rates $(27.7 \%$ vs. $6.1 \%, p<0.0001)$ than patients with normal renal function. ${ }^{21)}$ There are several leading explanations for why CKD is such a potent risk factor for adverse outcomes: 1) excess co-morbidities in CKD patients, including older age and diabetes, 2) underused end-organ protective strategies in CKD patients, or therapeutic nihilism, 3) excess toxicities from conventional therapies used including radio-contrast material and antithrombotic agents, and 4) the unique pathobiology of the CKD state, which includes intra-renal vasoconstriction when exposed to iodinated contrast agents. ${ }^{22)}$

Coronary stents have substantially improved the efficacy of percutaneous revascularization. DES has further reduced the rates of in-stent restenosis and repeat revascularization compared to BMS. ${ }^{23}$ Despite the widespread use of DES in treating CAD, which is prevalent among end-stage renal disease patients, the safety and efficacy of DES in this population have not been well-defined. The overall results of a meta-analysis, which included 869 patients in a study by Abdel-Latif et al. ${ }^{24)}$ indicate that DES use in these patients is safe and results in a modest, yet statistically significant reduction in adverse clinical events. Most important, the procedural success with DES was similar to that encountered with BMS. DES use significantly reduced the risk of TLR/TVR and MACE, but did not significantly influence the risk of all-cause mortality or MI. Hence, we included all CKD patients who received either BMS or any DES in this study cohort.

Prior studies by Halkin et al. ${ }^{25)}$ in dialysis patients, as well as by Zhang et al. ${ }^{26)}$ in patients with moderate renal insufficiency have found superiority of DES versus BMS in patients with CKD in the reduction of MACE in 1-2 years. A recent study by Barthelemy et al. ${ }^{27)}$ showed that the use of DES in non-selected patients with CKD appears at least as effective and safe as BMS at 1 year, although these patients carry a higher risk of restenosis. In our study, we found similar risk of MACE among the groups. PES group patients were worse than in the EES group. They were elder with higher incidence of past history of CAD, increased level of LDL-C and higher Killip classes. In addition, they had higher incidence of left main stem disease, ACC/AHA lesion type $C$, increased stent length, stent diameter, post procedure diameter stenosis, and a higher rate of in-hospital complications. However, multivariate Cox regression analysis, using significant baseline predictors, revealed no statistical differences in the incidence of 12-month MACE.

In general population, DES is associated with an increased risk of late stent thrombosis compared with BMS. However, we found similar incidence of stent thrombosis with BMS compared to other DES groups. This finding is notable since CKD has been described as a risk factor for stent thrombosis after DES implantation. ${ }^{28)}$ A possible explanation for this observation might be that the baseline endothelial dysfunction and inflammatory milieu in patients with CKD increases the risk of stent thrombosis to similar degrees with DES and BMS. ${ }^{29)}$ In this study, EES showed the lowest rate of cardiac death. EES is a newer generation DES and there have been many improvements in antiplatelet therapy, antihypertensive medications, and antidiabetic medications over the last few years. Decreased cardiac death could also be due to better stent technique with a different delivery system and different polymers. These improved immediate procedural success rates could have resulted in a lower cardiac death rate in the EES group.

The present study is limited due to its retrospective nature. Moreover, the study could have been under powered because of an added confounding effect from multiple types of stents. We did not study outcomes stratified by every stage of CKD and for patients on dialysis. Patients did not undergo follow-up stress test or coronary angiography; silent ischemia, and restenosis could have been under diagnosed. Patients enrolled into this study did not undergo a followup renal function test after $\mathrm{PCl}$, to rule out acute kidney injury.

In conclusion, the rate of cumulative MACE differed significantly among the stent groups (lowest in EES), mainly driven by the risk of cardiac death. However, after final adjustment in multivariate analysis and after propensity score adjustment, the same difference did not reach statistical significance. Therefore, we conclude that any of the 5 commonly used stents can be used to treat STEMI patients with CKD (eGFR $<60 \mathrm{~mL} / \mathrm{min} / 1.73 \mathrm{~m}^{2}$ ) undergoing primary $\mathrm{PCl}$, with a similar risk of 12-month MACE. Our observations may be influenced by selection bias, residual confounding, or may represent a true finding; at a minimum, this study has generated hypotheses that need to be further investigated through a long-term, randomized controlled study.

\section{Acknowledgments}

The authors are highly thankful to Dr. Sumera Ahmed for her help in statistical analysis and formulation of this manuscript. 


\section{Appendix}

Korea Acute Myocardial Infarction Registry Study Group of Korean Society of Cardiology.

Myung Ho Jeong, MD, Youngkeun Ahn, MD, Shung Chull Chae, MD, Jong Hyun Kim, MD, Seung Ho Hur, MD, Young Jo Kim, MD, In Whan Seong, MD, Dong Hoon Choi, MD, Jei Keon Chae, MD, Taek Jong Hong, MD, Jae Young Rhew, MD, Doo II Kim, MD, In Ho Chae, MD, Jung Han Yoon, MD, Bon Kwon Koo, MD, Byung Ok Kim, MD, Myoung Yong Lee, MD, Kee Sik Kim, MD, Jin Young Hwang, MD, Myeong Chan Cho, MD, Seok Kyu Oh, MD, Nae Hee Lee, MD, Kyoung Tae Jeong, MD, Seung Jea Tahk, MD, Jang Ho Bae, MD, Seung Woon Rha, MD, Keum Soo Park, MD, Chong Jin Kim, MD, Kyoo Rok Han, MD, Tae Hoon Ahn, MD, Moo Hyun Kim, MD, Ki Bae Seung, MD, Wook Sung Chung, MD, Ju Young Yang, MD, Chong Yun Rhim, MD, Hyeon Cheol Gwon, MD, Seong Wook Park, MD, Young Youp Koh, MD, Seung Jae Joo, MD, Soo Joong Kim, MD, Dong Kyu Jin, MD, Jin Man Cho, MD, Byung Ok Kim, MD, Sang-Wook Kim, MD, Jeong Kyung Kim, MD, Tae Ik Kim, MD, Deug Young Nah, MD, Si Hoon Park, MD, Sang Hyun Lee, MD, Seung Uk Lee, MD, Hang-Jae Chung, $M D$, Jang Hyun Cho, MD, Seung Won Jin, MD, Yang Soo Jang, MD, Jeong Gwan Cho, MD, and Seung Jung Park, MD

\section{References}

1. Lindner A, Charra B, Sherrard DJ, Scribner BH. Accelerated atherosclerosis in prolonged maintenance hemodialysis. N Engl J Med 1974;290: 697-701.

2. Dumaine $R$, Collet JP, Tanguy ML, et al. Prognostic significance of renal insufficiency in patients presenting with acute coronary syndrome (the Prospective Multicenter SYCOMORE study). Am J Cardiol 2004;94: 1543-7.

3. Manjunath $G$, Tighiouart $H$, Ibrahim $H$, et al. Level of kidney function as a risk factor for atherosclerotic cardiovascular outcomes in the community. J Am Coll Cardiol 2003;41:47-55.

4. Best PJ, Lennon $\mathrm{R}$, Ting $\mathrm{HH}$, et al. The impact of renal insufficiency on clinical outcomes in patients undergoing percutaneous coronary interventions. J Am Coll Cardiol 2002;39:1113-9.

5. Naidu SS, Selzer $F_{1}$ Jacobs $A$, et al. Renal insufficiency is an independent predictor of mortality after percutaneous coronary intervention. Am J Cardio/ 2003;92:1160-4.

6. Azar RR, Prpic R, Ho KK, et al. Impact of end-stage renal disease on clinical and angiographic outcomes after coronary stenting. Am J Cardiol 2000;86:485-9.

7. Best PJ, Berger PB, Davis BR, et al. Impact of mild or moderate chronic kidney disease on the frequency of restenosis: results from the PRESTO trial. J Am Coll Cardio/ 2004;44:1786-91.

8. Das $P_{1}$ Moliterno DJ, Charnigo $R$, et al. Impact of drug-eluting stents on outcomes of patients with end-stage renal disease undergoing percutaneous coronary revascularization. J Invasive Cardio/ 2006;18:405-8.

9. Lemos PA, Arampatzis $C A$, Hoye $A$, et al. Impact of baseline renal func- tion on mortality after percutaneous coronary intervention with sirolimus-eluting stents or bare metal stents. Am J Cardio/ 2005;95:167-72.

10. Charytan D, Kuntz RE. The exclusion of patients with chronic kidney disease from clinical trials in coronary artery disease. Kidney Int 2006; 70:2021-30.

11. Lee $K H_{1}$ Jeong $M H_{1} A h n Y K$, et al. Sex differences of the clinical characteristics and early management in the Korea Acute Myocardial Infarction Registry. Korean Circ J 2007;37:64-71.

12. Sim DS, Kim JH, Jeong MH. Differences in clinical outcomes between patients with ST-elevation versus non-ST-elevation acute myocardial infarction in Korea. Korean Circ J 2009;39:297-303.

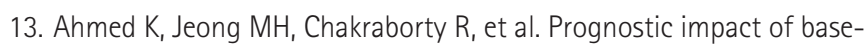
line high-sensitivity $\mathrm{C}$-reactive protein in patients with acute myocardial infarction undergoing percutaneous coronary intervention based on body mass index. Korean Circ J 2012;42:164-72.

14. National Kidney Foundation. K/DOOI clinical practice guidelines for chronic kidney disease: evaluation, classification, and stratification. Am J Kidney Dis 2002;39(2 Suppl 1):S1-266.

15. Apple FS, Wu AH, Jaffe AS. European Society of Cardiology and American College of Cardiology guidelines for redefinition of myocardial infarction: how to use existing assays clinically and for clinical trials. Am Heart J 2002;144:981-6.

16. Beattie JN, Soman SS, Sandberg KR, et al. Determinants of mortality after myocardial infarction in patients with advanced renal dysfunction. Am J Kidney Dis 2001;37:1191-200.

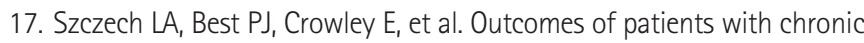
renal insufficiency in the bypass angioplasty revascularization investigation. Circulation 2002;105:2253-8.

18. Bonello L, De Labriolle A, Roy P, et al. Impact of optimal medical therapy and revascularization on outcome of patients with chronic kidney disease and on dialysis who presented with acute coronary syndrome. Am J Cardiol 2008;102:535-40.

19. Keeley EC, Kadakia R, Soman S, Borzak S, McCullough PA. Analysis of long-term survival after revascularization in patients with chronic kidney disease presenting with acute coronary syndromes. Am J Cardiol 2003;92:509-14.

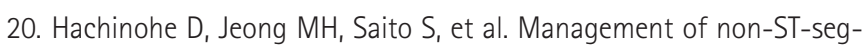
ment elevation acute myocardial infarction in patients with chronic kidney disease (from the Korea Acute Myocardial Infarction Registry). Am J Cardiol 2011;108:206-13.

21. Rubenstein MH, Harrell LC, Sheynberg BV, Schunkert $H_{1}$ Bazari $H_{1}$ Palacios IF. Are patients with renal failure good candidates for percutaneous coronary revascularization in the new device era? Circulation 2000; 102:2966-72.

22. McCullough PA. Cardiorenal risk: an important clinical intersection. Rev Cardiovasc Med 2002;3:71-6.

23. Kirtane AJ, Gupta A, lyengar S, et al. Safety and efficacy of drug-eluting and bare metal stents: comprehensive meta-analysis of randomized trials and observational studies. Circulation 2009;119:3198-206.

24. Abdel-Latif A, Mukherjee D, Mesgarzadeh P, Ziada KM. Drug-eluting stents in patients with end-stage renal disease: meta-analysis and systematic review of the literature. Catheter Cardiovasc Interv 2010;76: 942-8. 
25. Halkin A, Selzer F, Marroquin O, Laskey W, Detre K, Cohen H. Clinical outcomes following percutaneous coronary intervention with drugeluting vs. bare-metal stents in dialysis patients. J Invasive Cardio/2006; 18:577-83.

26. Zhang RY, Ni JW, Zhang JS, et al. Long term clinical outcomes in patients with moderate renal insufficiency undergoing stent based percutaneous coronary intervention. Chin Med J (Engl) 2006;119:1176-81.

27. Barthelemy O, Helft G, Le Feuvre C. One year clinical outcomes in pa- tients with chronic kidney disease treated by percutaneous coronary intervention with drug-eluting stent. Int J Cardiol 2011;147:307.

28. lakovou I, Schmidt T, Bonizzoni E, et al. Incidence, predictors, and outcome of thrombosis after successful implantation of drug-eluting stents. JAMA 2005;293:2126-30.

29. Shenoy C, Boura J, Orshaw P, Harjai KJ. Drug-eluting stents in patients with chronic kidney disease: a prospective registry study. PLoS One 2010;5:e15070. 